\title{
Enseñando a hacer entrevistas en investigación cualitativa: entre el oficio, la profesión y el arte ${ }^{1}$
}

\author{
ANALÍA MeO \\ Universidad de Buenos Aires \\ analiameo@fsoc.uba.ar \\ AlEJANDRA NAVARRO \\ Universidad de Buenos Aires \\ navarroalejandra@ymail.com
}

Recibido: 12.11 .2008

Aceptado: 26.05.2009

En el marco de la investigación cualitativa, la enseñanza de metodología está plagada de interrogantes, dudas y controversias. En estos tiempos de tormentas, de múltiples paradigmas epistemológicos, teóricos y metodológicos (Hammersley 1992; Bryman 2000; Denzin y Lincoln 2000; Guber 2004) muchas son las preguntas que atraviesan nuestro quehacer cotidiano: ¿qué entendemos por investigación cualitativa?, ¿es posible producir conocimiento acerca de lo social en tiempos de crisis de representación, autenticidad y legitimidad?, ¿cómo interpretamos el trabajo que realizamos?, ¿es un trabajo cercano al oficio, a la profesión o al arte? y, ¿de qué modo estas diferentes concepciones acerca de la investigación y del investigador/a impactan en la forma en que enseñamos?.

Estas preguntas no son nuevas. Sin embargo, como afirma Hammersley (2004: 549, traducción propia), la existencia de diversas formas de hacer investigación cualitativa exige tomas de posiciones respecto de «lo que se puede o no se puede, de lo que se debería y no debería» hacer cuando se enseña metodología. Al mismo tiempo, existen fuertes presiones dentro y fuera de la academia para definir las formas legítimas de hacer investigación, lo cual tiene implicancias en el modo en que los/as estudiantes son entrenados en las técnicas consi-

\footnotetext{
${ }^{1}$ Las autoras agradecen a los alumnos del curso «Haciendo entrevistas: fundamentos, principios y prácticas» por contribuir a la creación de un espacio de discusión colectivo, respetuoso y plural que nos permitió reflexionar críticamente sobre nuestra práctica docente. Asimismo, agradecen los constructivos comentarios de las dos personas que evaluaron este artículo.
}

EMPIRIA. Revista de Metodología de Ciencias Sociales. N.o 17, enero-junio, 2009, pp. 123-140. ISSN: $1139-5737$ 
deradas apropiadas para cumplir con los roles profesionales esperados (Hammersley 2004).

Este artículo se propone responder a la pregunta ¿cómo se enseña a hacer investigación utilizando entrevistas cualitativas? Para ello, se analiza una experiencia docente a nivel de posgrado en la Universidad de Buenos Aires (Argentina) durante el año académico 2008. El trabajo argumentará que la enseñanza del uso de entrevistas exige el despliegue de variadas instancias pedagógicas o interview practica (Kvale 2006), las cuales contribuyen a la apropiación práctica y reflexiva, por parte de los/as estudiantes, de un habitus de investigación que promueva su ejercicio ético y profesional.

Como toda experiencia pedagógica, el curso se fundó en concepciones específicas acerca de lo que es la investigación cualitativa, de su naturaleza, ventajas, límites y posibilidades; así como del rol del investigador, de sus competencias o habilidades fundamentales y de sus responsabilidades respecto de los sujetos investigados, de su comunidad académica y de la sociedad en general (Jenkins 1995; Hammersley 2004; Kvale 2006). En la primera sección de este trabajo explicitaremos cuál es la concepción del rol del/a investigador/a cualitativo/a que nutrió la selección de los contenidos y el diseño pedagógico de este curso de posgrado. Argumentaremos que el trabajo de los/as investigadores cualitativos debe ser entendido como una profesión que también tiene aspectos creativos y propios de un oficio. Seguidamente caracterizaremos el curso de posgrado, su población, así como diversos tipos de interview practica que diseñamos para inculcar un habitus de investigación sociológico orientado a la generación de conocimiento que se interroga permanentemente sobre las condiciones de su producción, sus supuestos teóricos, y sobre su legitimidad y límites. Fundamentaremos nuestro análisis en observaciones de clase, registros de las discusiones realizadas, ejercicios resueltos y una encuesta de evaluación aplicada al finalizar el curso.

\section{EL INVESTIGADOR CUALITATIVO: ENTRE EL OFICIO, LA PROFESIÓN Y EL ARTE}

La discusión acerca de la naturaleza de la investigación cualitativa y del rol de la persona que la lleva a cabo no es nueva. De hecho, ha atravesado la historia y el presente del paradigma cualitativo (Denzin y Lincoln 2000; Vidich y Lyman 1994; Schwandt 2000). En los países anglosajones, la pluralidad epistemológica, teórica y metodológica caracteriza al quehacer de las comunidades de investigadores cualitativos que trabajan en diferentes campos disciplinares (tales como antropología, educación, sociología, psicología, ciencias de la comunicación, trabajo social y estudios culturales).

En los distintos contextos nacionales y en sus campos y sub-campos académicos, diferentes comunidades de investigadores que se auto-definen como cualitativos adscriben a variados y hasta antagónicos principios ontológicos y epis- 
temológicos que fundan el tipo de conocimiento que producen, la forma en que lo construyen y la forma privilegiada en que lo representan (Denzin y Lincoln 2000). En Argentina, también hay evidencia de un emergente pluralismo metodológico (en un sentido amplio) que asume particularidades y reconoce excepciones en distintos campos académicos (Vasilachis de Gialdino 2007).

A pesar de las diferentes trayectorias temáticas, teóricas y de estrategias metodológicas recorridas por las autoras, compartimos algunas miradas fundamentales acerca de la naturaleza de la investigación cualitativa, de sus fines y formas legítimas de producir conocimiento. En primer lugar, entendemos que «los investigadores cualitativos estudian las cosas en su medio natural, intentando darle sentido, o interpretar, el fenómeno en los términos que la gente les otorga» (Denzin y Lincoln 2005: 3, traducción propia). En otras palabras, consideramos que a partir de la investigación cualitativa nos proponemos entender los marcos de referencia y de sentido de las personas estudiadas reconociendo el carácter situado de sus prácticas y discursos así como la naturaleza dinámica y procesual de la realidad social (Bryman 2000). En segundo lugar, entendemos que la investigación se propone producir conocimiento acerca de lo social. En este sentido, supone una ontología realista (o crítico realista) y una epistemología que reconoce el carácter construido, social y localizado del conocimiento que produce $\mathrm{y}$, por tanto, que reanuncia al ideal naturalista propio de la fase modernista del paradigma cualitativo (Denzin y Lincoln 2005). Por esta razón, se debe ser reflexivo y examinar las condiciones sociales, políticas, éticas y académicas de las condiciones en las que produce conocimiento (Bourdieu y Wacquant 1995; Jenkins 1995; Hertz 1997; Macbeth 2001; Maton 2003). En este trabajo, entenderemos a la reflexividad como un concepto multidimensional que refiere tanto a la objetivación de la práctica investigativa como a su examen crítico a la luz de diferentes lentes. La reflexividad, en estos términos, es interpretada como fundamental para dar respuestas (tanto individuales como colectivas) a las profundas crisis de representación, de legitimidad y de la praxis que desde la década del '70 caracteriza a las ciencias sociales (Denzin y Lincoln 2005).

Para avanzar en la definición de un modelo de investigación cualitativa que sea capaz de de orientar nuestra tarea docente en un sentido reflexivo utilizaremos críticamente el análisis de Hammersley (2004). Este autor identifica cuatro modelos de investigación cualitativa que subyacen a diferentes formas de abordar la enseñanza de metodología de la investigación: la investigación como procedimiento, la investigación como oficio (craft), la investigación como profesión, y la investigación como arte. Entender a la investigación como procedimiento implica asociarla con una serie de técnicas que deben ser aprendidas para su posterior aplicación en investigaciones concretas. Siguiendo a este autor, esta forma de entender a la investigación social está muy alejada de la práctica de la investigación y de las visiones de los otros tres modelos. A continuación examinaremos estos tres modelos y argumentaremos que, si bien no son totalmente incompatibles, suponen diferentes concepciones acerca de las habilidades (skills) fundamentales del investigador cualitativo y de las formas que éstas deben adquirirse.

EMPIRIA. Revista de Metodología de Ciencias Sociales. N.o 17, enero-junio, 2009, pp. 123-140. ISSN: 1139-5737 
Concebir a la investigación como un oficio implica destacar su carácter práctico, colectivo e individual. En palabras de Kvale (2006: 3, traducción propia), «es una forma de producción que exige habilidad artística y destreza; supone agudeza personal y se adquiere a partir de mucho entrenamiento y práctica». Asimismo, este modelo centra el proceso de enseñanza-aprendizaje en la relación docente-estudiante y en la transmisión directa y personal de la experiencia y el conocimiento especializado a través de ejercicios prácticos (Becker 1998; Hammersley 2004; Kvale 2006).

Por otro lado, entender a la investigación como profesión implica, entre otras $\cos ^{2}$, reconocer la autonomía de los/as profesionales en el ejercicio de su ocupación. Esta autonomía es limitada tanto «en términos individuales como colectivos» (Hammersley 2004: 553, traducción propia) y restringe el accionar a todo aquello que puede justificarse como necesario para el cumplimiento de los fines profesionales. La enseñanza de la investigación cualitativa, para este modelo, debe inculcar una responsabilidad ética en los/as estudiantes en un contexto en donde no existen consensos acerca de lo que suponen estos estándares éticos. Asimismo, entender a la investigación cualitativa como una profesión supone altos niveles de reflexividad (tanto individual como colectiva) que apuntan a explicitar cómo el conocimiento se produce y cuáles son los efectos que ese conocimiento tiene sobre los sujetos investigados, sobre la profesión y la sociedad en general (Guber 2004; Hammersley 2004; Bourdieu y Wacquant 1995). Desde el punto de vista pedagógico, esto implica la adquisición de recursos cognitivos que permitan interrogar y compartir prácticas de investigación (Hammersley 2004; Sautu y Wainerman 1997). Esta reflexividad, al igual que la autonomía profesional, está limitada al compromiso de la profesión con la producción de conocimiento. En este sentido, en la visión profesional de la investigación, los/as investigadores/as no deben interrogar todos los supuestos ni deben repensar o recrear los fines de su tarea profesional. En palabras de Hammersley (2004), la diferencia central entre este modelo y el que interpreta a la investigación cualitativa como un oficio es:

«una profesión requiere mayor reflexividad por parte del que la ejerce, y por lo tanto por parte de los que ingresan a ella, que un oficio (craft). Sin embargo, la reflexividad no es relevante en sí misma sino sólo en la medida que facilita el cumplimiento de los fines de la ocupación»

(Hammersley 2004: 554, traducción propia)

Hammersley (2004) también examina al modelo de investigación entendido como arte, el cual es definido a partir de su carácter «creativo, nuevo, sorprendente, o provocador» (555, traducción propia). En esta interpretación, la investigación como arte supone ir más allá de la noción de oficio y se opone a la de

${ }^{2}$ El concepto de profesional refiere también al manejo de un conocimiento experto así como al reconocimiento o status social que su ejercicio acarrea.

EMPIRIA. Revista de Metodología de Ciencias Sociales. N.o 17, enero-junio, 2009, pp. 123-140. ISSN: $1139-5737$ 
profesión. Hammersley (2004) asocia esta visión a los autores Denzin y Lincoln (2005) y argumenta que éstos sostienen que la investigación cualitativa es similar al arte contemporáneo en donde lo central es la imaginación y la libertad respecto «de los límites de la realidad» (Hammersley 2004: 555, traducción propia). En términos pedagógicos, este tipo de aproximación supone la persona que investiga, en tanto bricoleur, es un auto-didacta. En todo caso, debería enseñarse a cuestionar todos los supuestos de su tarea y, así, desplegar una reflexividad fundamentalmente negativa.

A diferencia del análisis de Hammersley (2004), consideramos que el modelo de investigación que debe orientar nuestras prácticas docentes debe integrar, antes que disociar, diferentes elementos de estos tres modelos de investigación cualitativa. Asimismo, consideramos que es necesario redefinir el tercer modelo identificado por el autor.

Desde nuestra perspectiva, el investigador cualitativo es entendido como un profesional que lleva adelante una tarea con características propias de un oficio que se nutre también de la creatividad y la imaginación -similar a la que despliegan los artistas aunque con sus propias formas y criterios de comunicación (Nisbet 1963). Desde el punto de vista pedagógico, este modelo supondrá: i) la transmisión de los «trucos del oficio» (tricks of the trade) (Becker 1998); ii) la socialización en una reflexividad mulitidimensional (Reay 1996; Macbeth 2001; Maton 2003; Pillow 2003; Hammersley 2004); y iii) la imaginación sociológica (Mills 1994). Esta forma de entender la investigación cualitativa combina aspectos centrales propios de los oficios y profesiones, identificados por Hammersley (2004), y elementos de un modelo alternativo la investigación como arte - centrado en Nisbet (1923) que integra el concepto de imaginación sociológica de Mills (1959).

Nuestro modelo de investigación cualitativa reconoce que nuestra tarea es un oficio. Entre ellos, de fundamental importancia para pensar la enseñanza, la relevancia de los «trucos» del oficio -tricks of the trade en términos de Becker (1998). Estos trucos refieren, por un lado, a conocimientos acumulados colectivamente por los investigadores que sirven para solucionar problemas recurrentes y comunes a los estudios cualitativos. Así, por ejemplo, transcribir las entrevistas siguiendo códigos de transcripción pre-definidos que enriquezcan el posterior análisis puede ser interpretado como un truco que los investigadores más experimentados conocen más por viejos y por experimentados que por sabios. Por otro lado, Becker (1998) argumenta que también es necesario transmitir otro tipo de trucos. Éstos antes que simplificar la tarea de investigación la complican exigiendo una constante interrogación respecto de la autenticidad del conocimiento producido.

El modelo de investigación que orientó nuestra práctica docente reconoce también que los/as investigadores/as pertenecen a asociaciones profesionales (nacionales y/o internacionales) y, con ello, deben responder a sus exigencias y marcos normativo-legales y éticos (Meo 2009). Así, siguiendo a Hammersley (2004), este modelo reconoce a la producción del conocimiento como la finali-

EMPIRIA. Revista de Metodología de Ciencias Sociales. N.o 17, enero-junio, 2009, pp. 123-140. ISSN: 1139-5737 
dad central de la profesión, y demanda altos niveles de reflexividad en torno a las condiciones (sociales, políticas, éticas y epistémicas) en las que se genera.

Finalmente este modelo de investigación incorpora una dimensión cercana al arte, a la imaginación y a la creación como parte del quehacer investigativo. Como el artista, la persona que investiga se interesa en entender su mundo, trabaja con materiales empíricos y busca descubrir relaciones significativas (Nisbet 1963). Como la creación de una obra de arte, el descubrimiento científico supone una gran creatividad que va mucho más allá de la aplicación de un método para la resolución de un problema. Nisbet nos recuerda que sin esta creatividad, no habría hipótesis a ser testeadas por diferentes medios. En sus palabras: «cualquier cosa que reduzca el campo de la experiencia y la imaginación, que disminuya las fuentes de inspiración, que vuelva rutinario a la mente inteligente, debe ser vista con sospecha» (1963: 155, traducción propia). Sin embargo, la investigación difiere del arte, la creatividad y la imaginación se transforman en ciencia sólo cuando siguen una serie de criterios y formas de comunicación propios de las comunidades científicas, las cuales difieren notablemente de las del arte.

Consideramos que pensar a la investigación cualitativa como arte puede ser también enriquecido por el concepto de imaginación sociológica propuesto por Mills (1994). En sus palabras:

«La imaginación sociológica permite a su poseedor comprender el escenario histórico más amplio en cuanto a su significado para la vida interior y para la trayectoria exterior de diversidad de individuos. Ella le permite tener en cuenta cómo los individuos, en el tumulto de su experiencia cotidiana, son con frecuencia falsamente -conscientes de sus posiciones sociales. En aquel tumulto se busca la trama de la sociedad moderna, y dentro de esa trama se formulan las psicologías de una diversidad de hombres y mujeres. Por tales medios, el malestar personal de los individuos se enfoca sobre inquietudes explícitas y la indiferencia de los públicos se convierte en interés por las cuestiones públicas.» (Mills 1994: 25)

En este sentido, esta imaginación permite pasar de un plano a otro de análisis con el propósito de dar sentido al mundo y articular aspectos de maneras novedosas. Sin duda, este tipo de imaginación puede cultivarse y, en palabras de Mills (1994) es la que separa al mero técnico del investigador social. En nuestra perspectiva, la imaginación sociológica debe desplegarse en relación al fenómeno estudiado pero también al propio quehacer investigativo. Así, la imaginación sociológica se aproxima a nociones amplias de reflexividad que apelan a localizar la tarea del investigador (sus aspectos privados e íntimos) en el campo más amplio de la disciplina y de las ciencias sociales. Así, la imaginación sociológica orienta el análisis de los datos y expande nuestros horizontes interpretativos pero también vincula las tareas cotidianas del investigador con cuestiones públicas que conciernen a la comunidad académica a la que pertenece.

Hasta el momento hemos identificado las características centrales del modelo de investigación que guía nuestra práctica de investigación y que orientó el di-

EMPIRIA. Revista de Metodología de Ciencias Sociales. N.o 17, enero-junio, 2009, pp. 123-140. ISSN: $1139-5737$ 
seño curricular y pedagógico del curso que dictamos sobre el uso de entrevistas. La sección siguiente caracterizará esa experiencia y argumentará que las diferentes instancias de interview practica que diseñamos favorecieron la adquisición práctica del oficio y de la profesión reflexiva e imaginativa que supone el hacer investigación cualitativa.

\section{SE HACE CAMINO AL ANDAR: LA ENSEÑANZA DEL USO DE ENTREVISTAS EN INVESTIGACIÓN SOCIAL}

El seminario de posgrado se propuso contribuir a la formación de estudiantes de doctorado reflexivos capaces de usar críticamente la entrevista como técnica de investigación de la realidad social. El objetivo central era brindarles herramientas teórico-metodológicas para poder desarrollar una investigación que por su objetivo requería de la utilización de entrevistas. Partimos del supuesto de que no es posible aprender a hacer investigación y a hacer entrevistas sin atravesar por esa experiencia, conjuntamente con el ejercicio continuo de pensarse y pensar cada situación que se presente a lo largo del proceso de investigación. Práctica y reflexión se transformaron en dos ideas rectoras del curso, tal como flexibilidad y emergencia lo son para los diseños cualitativos en lo que se imbrican las entrevistas (Maxwell 1996).

Tal como se explicitó en la introducción, el seminario se desarrolló en el marco del Doctorado ${ }^{3}$ en Ciencias Sociales de la Universidad de Buenos Aires. Este doctorado se caracteriza por promover una formación adaptada a las necesidades individuales de los/as estudiantes. Éstos diseñan sus trayectos educativos a partir de las características de sus propuestas de tesis, de su experiencia previa de investigación y de las sugerencias de la Comisión de Doctorado. Para ser aceptado por el Doctorado, los postulantes deben presentar y defender una propuesta de tesis frente a dos jurados/miembros de la Comisión de Doctorado.

Con respecto al seminario, el mismo se desarrolló en diez sesiones de tres horas cada una y se organizó en torno a tres tipos de encuentros. El primero consistió en clases teóricas en las cuales las docentes presentaban un tema. El segundo tipo de sesiones, daba cuenta de lo que Kvale (2006) llama interview práctica e implicó la implementación de distintas situaciones pedagógicas (que llamamos ejercicios y guías prácticas), las cuales pusieron en juego los saberes y habilidades (teóricos y metodológicos) de los/as estudiantes. Ambos dispositivos (ejercicios y guías) estaban relacionados con la temática que presentaban las docentes y sus respectivas consignas se entregaban una semana antes para su resolución individual (en el caso de los ejercicios) y su discusión grupal y puesta en común (en el caso de las guías). Asimismo, los contenidos de ambas instancias pedagógicas estaban articulados, ya que la guía práctica retomaba aspectos que los/as participantes debieron haber examinado individualmente para resolver el

\footnotetext{
${ }^{3}$ Este doctorado fue creado en 1996 bajo la Resolución de Creación Nro 4883/96.
} 
ejercicio correspondiente. Por último, las tutorías constituyeron el tercer tipo de encuentro implementado en el seminario. Las mismas brindaron la posibilidad de un intercambio cara a cara de los/as participantes con las docentes. Estos encuentros se organizaron a partir de un temario o lista de preguntas presentadas con antelación a las docentes. Las preguntas debían estar focalizadas en la temática del seminario, esto es, el uso de las entrevistas en el marco de la investigación social. Un poco más de la mitad del grupo participó en este espacio. Según la encuesta de evaluación del seminario, la participación en las tutorías respondió a la búsqueda de orientación y resolución de situaciones problemáticas a partir de la escucha de otra mirada ${ }^{4}$. Debido a razones de espacio, en esta sección sólo analizaremos las interview practica, los ejercicios y guías prácticas.

Con respecto al currículo del seminario, el temario seleccionado se proponía discutir diferentes cuestiones relacionadas con el proceso de investigación y la utilización de entrevistas para recoger la evidencia empírica. Los temas trabajados y discutidos críticamente fueron: i) los supuestos epistemológicos y metodológicos de la entrevista en el marco del paradigma cualitativo; ii) el concepto de reflexividad y el rol del/a investigador/a en el proceso de investigación; iii) las consideraciones e implicancias éticas de las investigaciones; iv) los criterios de selección de los «casos» a entrevistar; v) la construcción de la guía de entrevista y la situación de entrevista; y v) la sistematización, análisis e interpretación del material cualitativo.

Dadas las características formales del Doctorado arriba descriptas, los/as participantes del seminario ${ }^{5}$ tenían una propuesta de tesis (más o menos desarrollada según el caso) al iniciar el seminario, a partir de la cual se trabajó en cada uno de los encuentros. La mayoría eran estudiantes de doctorado y sólo tres habían culminado una maestría. El grupo en su totalidad trabajaba desde una perspectiva cualitativa pero dentro de corrientes de pensamiento distintas; y realizaba su trabajo de campo al mismo tiempo que cursaba el seminario. ${ }^{6}$ Sin embargo, sus trayectorias educativas e inserciones disciplinares así como sus perspectivas y temáticas se caracterizaron por su diversidad. Así, por ejemplo, si bien su formación de grado era en ciencias sociales y/o humanidades, la mitad eran de sociología, seguidos por egresados de ciencias políticas, antropología, psicología, ciencias de la educación, geografía y filosofía.

Luego de describir las características generales del seminario, su curriculum y su población, en la sección siguiente, vamos a analizar dos tipos de interview practica: los ejercicios y las guías prácticas.

\footnotetext{
${ }^{4}$ Este espacio individual de diálogo resultó óptimo para discutir situaciones puntuales que los estudiantes presentaban por lo general como problemáticas. La evaluación fue muy buena y respondió a las expectativas de docentes y estudiantes de encontrar respuestas, no recetas, a las situaciones presentadas.

${ }^{5}$ En la cohorte 2008 del curso participaron 19 alumnos (5 varones y 14 mujeres). Doce de ellos tenían entre 26 y 30 años. Cuatro estudiantes tenían entre 31 y 34 años y sólo uno era mayor de 50.

${ }^{6}$ Éste fue uno de los requisitos para inscribirse al seminario.
} 


\section{Interview practica: entre la reflexividad individual y la reflexividad colectiva}

A continuación examinaremos cómo los ejercicios y las guías contribuyeron a que los/as estudiantes se apropiaran de modos de objetivar la propia práctica investigativa que fomentaron la imaginación sociológica. Con este propósito utilizaremos información tomada de distintos tipos de fuentes: notas de observación de clase de las docentes, ejercicios individuales resueltos por los alumnos, y una encuesta de evaluación aplicada al grupo el último día de clases. Las notas de campo fueron registradas alternativamente por cada una de las docentes durante y después de cada encuentro. En ellas se incluyó información acerca de las discusiones en pequeños grupos como generales. La decisión de incluir una encuesta para indagar las características socio-demográficas de los participantes y evaluar sus opiniones en relación al seminario se fundamentó en que nos permitía recoger información en un único encuentro y así poder procesarla rápida y económicamente. La encuesta incluyó una batería de preguntas referidas a distintas dimensiones: características socio-demográficas y educativas; razones de la elección del seminario; expectativas en relación al seminario; evaluación de la utilidad de cada una de las instancias pedagógicas (ejercicio individual, ejercicios grupales y tutorías) en relación a su proyecto de tesis y de su formación como investigadores/as; y la relevancia de la bibliografía y de las sesiones teóricas.

Durante la cursada los/as estudiantes tuvieron que realizar nueve ejercicios individuales referidos a las diferentes etapas del proceso de investigación (desde la inscripción de sus investigaciones en el paradigma cualitativo hasta el análisis de los datos). Las consignas de los ejercicios fueron diseñadas con el propósito de que cada estudiante se interrogara acerca de diversos aspectos del proceso de sus propias investigaciones, como por ejemplo: la formulación de los objetivos de investigación; los supuestos epistemológicos, teóricos y metodológicos del estudio; la selección de los casos; la situación de entrevista y formulación de memos; el rol de sus identidades sociales en la producción y análisis de datos; la dimensión ética de la investigación; y la sistematización y análisis de la información.

En la encuesta de evaluación aplicada al final de la cursada, el grupo en su totalidad afirmó que los ejercicios les resultaron muy útiles o útiles, destacando que los mismos los obligaron a reflexionar sobre sus trabajos de investigación, repensar aspectos del mismo y poner en marcha el trabajo de campo.

«Considero que fueron muy útiles porque me llevaron a redefinir aspectos concretos de mi planteo de investigación» (Encuesta 1)

«Gracias a los ejercicios me encontré diseñando una guía de entrevista, haciendo efectivas dos entrevistas y trabajando con sus transcripciones, algo que desconocía por completo antes del seminario» (Encuesta 2) 
«Me permitieron pensar clase a clase no sólo sobre los textos sino sobre mi trabajo de tesis. En definitiva lo que espero de un seminario de doctorado» (Encuesta 9)

«Fueron espacios de reflexión sobre mi tesis; no siempre uno tiene esa oportunidad de un modo pedagógico» (Encuesta 11).

«Me obligaron a pensar dimensiones que no había tenido en cuenta y me sirvieron para chequear si había entendido o no los conceptos» (Encuesta 12).

Los/as estudiantes destacaron que los ejercicios fueron disparadores para poner en acción lo discutido en los teóricos, para repensar sus investigaciones y la interacción de sus distintos componentes. Operaron como un recurso pedagógico que sirvió para transmitir ciertas orientaciones, disposiciones, formas de entender el proceso de investigación y el trabajo de campo. De esta manera, actuaron como dispositivos para transmitir una práctica de investigación y para socializar al grupo en lo que Becker (1998) define «trucos» de la investigación. El primer tipo de «trucos», como vimos, implica compartir soluciones a problemas comunes a toda investigación cualitativa (como por ejemplo, los consejos asociados al desarrollo de la situación de entrevista). El segundo tipo de «truco» refiere a las actividades implementadas para desfamiliarizar la tarea de investigación (por ejemplo, interrogarse cotidianamente sobre el quehacer investigativo con el objetivo de mejorarlo). En otras palabras, los ejercicios fueron actividades orientadas a la práctica (implicaban un hacer específico) y suponían diferentes niveles de reflexividad en torno a su rol de investigadores/as (Hertz 1997; Macbeth 2001; Maton 2003).

Como ilustración de los ejercicios trabajados con los/as estudiantes, analizaremos el que refiere a la «situación de entrevista» Éste implicaba la escritura de diferentes memos ${ }^{7}$ que respondieran a cada uno de las siguientes consignas:

1. Descripción del lugar dónde se realizó la entrevista, cuándo se hizo, cómo se sintió , y cómo se relacionó con la persona entrevistada

2. Auto-evaluación utilizando los criterios de Kvale (1996: 148-149): i) informada, ii) estructurada, iii) clara, iv) considerada, v) sensible, vi) abierta, vii) con dirección (steering), viii) crítica, ix) remembering, y x) interpretativa.

3. Auto-evaluación de cómo condujeron la entrevista y cómo se sintieron en el proceso, identificando dificultades y problemas para conducir y para llevar adelante la guía de la entrevista.

4. Auto-evaluación escrita de la guía de entrevistas utilizada en relación a los objetivos de la investigación y de la calidad de los datos producidos.

\footnotetext{
${ }^{7}$ Este tipo de instrumento de registro y análisis ha sido ampliamente trabajado por Glaser y Strauss (1967).
}

EMPIRIA. Revista de Metodología de Ciencias Sociales. N. ${ }^{\circ}$ 17, enero-junio, 2009, pp. 123-140. ISSN: 1139-5737 
Cada una de estas consignas exigía desnaturalizar una situación de entrevista. En cuanto a la resolución de los ejercicios, en primer lugar, los/as estudiantes objetivaron la situación de intercambio con la persona entrevistada. Reflexionaron acerca del clima de la entrevista, los diferentes momentos de la interacción, el tipo de interacción, y sus sentimientos. Escribir sobre la situación de entrevista les permitió un primer distanciamiento de su práctica en el cual exploraron, con distintos estilos y énfasis, el «cómo» se hace investigación (Hammersley 2004). En una minoría de casos, este memo de entrevista también los permitió avanzar en el análisis de sus datos. En segundo lugar, cada estudiante se autoevaluó como entrevistador/a siguiendo una serie de criterios propuestos por Kvale (1996). Éstos apuntan a examinar diferentes características de la interacción con los/as entrevistados/as así como con los objetivos de la investigación. Esta consigna contribuyó a la realización de un examen de la calidad de los vínculos que establecieron con sus informantes. En la investigación cualitativa, siguiendo a Coffey (1999), la calidad de las relaciones sociales es central a la hora de evaluar la calidad de los datos producidos o, en otras palabras, la autenticidad de los hallazgos. En tercer lugar, los/as estudiantes centraron su análisis en cómo llevaron adelante la situación de entrevista. Aquí, la mirada estaba puesta en los obstáculos y las dificultades, lo cual exigió un mayor nivel de reflexividad y supuso la generación de hipótesis interpretativas que trataran de explicar el por qué el diálogo no fue cómo se esperaba. Nuevamente, se les pedía que examinaran su rol como instrumentos de investigación (research instrument) (Kvale 2006; Hamersley 2004). Finalmente, se solicitaba que se evaluara a la guía de entrevista utilizada a la luz de la interacción concreta. Aquí, los/as estudiantes reflexionaron en torno a lo que habían logrado en términos de los objetivos de la investigación e identificaron dimensiones o aspectos emergentes que deberían incluir en posteriores entrevistas. La mayoría propuso cambios a sus guías (ya sea incluyendo nuevas preguntas, reformulando o eliminando otras).

Este interview practicum (Kvale 2006) resulto útil en dos sentidos. Por un lado, pensar y pensarse en esa situación repercutió directamente sobre otras instancias del diseño, desde la reformulación de parte de los objetivos a, sobre todo, la re-elaboración de la guía de entrevista y la consideración de otros posibles casos para entrevistar. Esto es concordante con las características de los diseños cualitativos, flexibles y emergentes, cuya reelaboración se da en el marco de la práctica (Maxwell 1996). Por otro lado, la elaboración de memos de entrevista visualizó que somos parte de ese proceso, que la entrevista no es una sucesión de preguntas y respuestas sino una coproducción discursiva que debería estar acompañada por toda una reflexión contextual de la misma (Gubrium y Holstein 2002; Hammersley y Atkinson 1995; Kvale 1996, 2006).

Esta tarea de autorreflexión (reflexividad individual), tal como mencionamos antes, estuvo acompañada por la discusión grupal de temáticas similares y por la puesta en común de las experiencias. La discusión grupal organizada en torno a guías prácticas se proponía promover la capacidad de articular argumentaciones y defenderlas frente a pares e investigadores más experimentados (Hammersley

EMPIRIA. Revista de Metodología de Ciencias Sociales. N.o 17, enero-junio, 2009, pp. 123-140. ISSN: 1139-5737 
2004; Lahire 2006). Como vimos en la primera sección, esta capacidad dialógica, crítica y constructiva de los miembros de la comunidad académica es central dentro del modelo profesional de la investigación cualitativa.

En cuanto a las ocho guías prácticas, como ya adelantamos, la gran mayoría retomaba temas trabajados en las clases teóricas y problematizados en los ejercicios. Las guías se proponían promover discusiones en pequeños grupos que luego eran puestas en común y sistematizadas por las docentes. Sus consignas incluían una dimensión comparativa de las experiencias y reflexiones del grupo. Así, las guías propiciaban el diálogo y el intercambio en torno a diversos materiales como investigaciones; bibliografía sobre epistemología y metodología; y notas de campo, entrevistas, y memos de distintos tipo generados por los alumnos. Para los/as estudiantes, la discusión grupal implicó: i) la objetivación de diversos aspectos del proceso de investigación, ii) una auto-evaluación del modo en que sus identidades sociales influyeron en el antes, el durante y el después de las entrevistas, iii) el hacer «públicos» estos ejercicios de objetivación, iv) justificar sus decisiones ante sus pares, y, por último, v) interrogar a sus compañeros respecto de sus ejercicios y, al hacerlo, ayudarlos a clarificar situaciones, conceptos, y/o decisiones y, fundamentalmente, a hacerse más preguntas. Las guías promovieron también la imaginación sociológica, posibilitando que los/as estudiantes se movieran de un plano a otro del análisis - de lo individual a lo grupal/colectivo- y favorecieron la inscripción de las experiencias «privadas» en el ámbito de lo público y, al hacerlo, mayores niveles de reflexividad.

En relación a esta experiencia pedagógica, la mayoría expresó la importancia del intercambio, básicamente por dos motivos: i) compartir experiencias con colegas que están atravesando situaciones similares; y ii) exponerse a la visión de los otros.

El compartir la experiencia con pares permitió salir del trabajo solitario propio del becario y de muchos investigadores. El siguiente testimonio resulta muy elocuente en relación a esto «... se quebró la soledad...» (Encuesta 3 ). En ese «compartir» emergieron sugerencias y aportes de los pares quienes apelaban a sus propias realidades para aconsejar. Un miembro del grupo señala,

«las sesiones de discusión me permitieron compartir con mis compañeros de seminario y con las profesoras los temas discutidos en cada clase. De cada una de las sesiones me llevo información que no la hubiera tenido en cuenta si no se realizaban» (Encuesta 8$)$.

En relación a estas discusiones grupales, fue posible identificar en varios relatos la redefinición de aspectos concretos de los trabajos de investigación, «...las sesiones me permitieron repensar la guía logrando una mayor adecuación entre ésta y mis objetivos» (Encuesta 9); «...surgieron reflexiones y experiencias similares, preguntas, inquietudes, resultaron disparadoras de aclaraciones muy interesantes» (Encuesta 11). Vemos como el diálogo y el intercambio 
promovió una dimensión colectiva de reflexividad que enriqueció la tarea de investigación.

En relación al intercambio y la visión de pares y colegas, los/as estudiantes señalaron que esta instancia les permitió «... poner a prueba el trabajo de uno...» (Encuesta 4); así como «.... validar mis futuros resultados...» (Encuesta 9); y «... defender lo que pensaba...» (Encuesta 14). Como señala Sautu (1997), la presentación de nuestras ideas y trabajos resulta central para la conformación y crecimiento del conocimiento en el marco de una comunidad académica. A partir de nuestros registros de sesiones, resultó evidente que la discusión y el «aprendizaje grupal» contribuyeron a repensar aspectos claves del proceso de investigación de cada estudiante, así como adquirir mayor seguridad para afrontar los desafíos del trabajo de campo.

Como ejemplo de guía práctica analizaremos la de la situación de entrevista, la cual se discutió en la misma clase en que los alumnos debían entregar el correspondiente ejercicio. Esta guía solicitaba:

En grupos (máximo 3 personas),

1. Discutir los memos de la situación de entrevista (20 minutos).

a. Comparen el tipo de información incluida y evalúen la relevancia para entender la interacción de los participantes.

b. Evalúen si podría haberse incluido otro tipo de información o qué aspectos valdría la pena incluir en futuros memos.

2. Discutir y comparar los resultados de cada una de las auto-evaluaciones (40 minutos).

a. ¿Cómo se sintieron durante la situación de entrevista? ¿Momentos de incomodidad? ¿puntos de inflexión en la dinámica de la entrevista?

b. ¿Cómo iniciaron la entrevista?

c. ¿Cómo cerró la entrevista? ¿Por qué la cerró en ese momento?

d. Identifiquen los problemas comunes y específicos de cada situaciónede entrevista.

a. Identifiquen estrategias para superar los problemas u obstáculos encontrados. ¿Qué cosas se podrían hacer diferente en la siguiente entrevista?

3. Discutir la auto-evaluación de cada una de las guías de entrevistas (25 minutos)

a. ¿en qué medida se respondieron las preguntas de la guía? ¿qué cosas faltaron? ¿por qué?

b. ¿qué preguntas valdría la pena incluir en la próxima entrevista? ¿para qué?

c. ¿reformularían la guía? ¿por qué? ¿cómo?

Esta guía propone discutir en detalle los aspectos centrales abordados por el correspondiente ejercicio. Por ejemplo, para dar cuenta del primer punto los/as estudiantes comentaron primero el contenido de sus memos de la situación de 
entrevista. ${ }^{8}$ Aquí examinaron críticamente si los registros eran útiles para examinar la relación y el clima que se generó entre la persona que entrevistaba y las que fueron entrevistadas. En este diálogo, la lectura y/o relato respecto de los memos promovió ricas conversaciones en torno al tipo de información que vale la pena registrar y a los beneficios que este tipo de instrumento conlleva (tanto respecto al trabajo de campo como al posterior tratamiento de la información). Cuando discutieron el segundo punto, los miembros del seminario pudieron compartir aspectos «privados» de su experiencia de investigación. Este espacio de diálogo les exigió poner en palabras incertidumbres, dudas, y críticas respecto de la propia tarea, a la vez que aprehender el carácter común de muchas de sus experiencias y dificultades. Así, pudieron verse en el trabajo de los otros y ampliar sus propios criterios para examinar cómo llevaron adelante sus entrevistas.

Luego de la discusión en pequeños grupos, las docentes intentaron identificar aspectos comunes y particulares de las experiencias y perspectivas de los/as estudiantes (con desiguales niveles de éxito) que emergieron en la discusión. En una minoría de sesiones (al principio de la cursada) fue complicado sistematizar lo discutido en grupos. En esta primera etapa del taller, fue difícil objetivar la dimensión grupal o colectiva de la reflexión. Así, las discusiones - por momentos - se focalizaron en situaciones problemáticas puntuales que emergieron en algunas investigaciones. A partir de la cuarta sesión (y luego de discusiones en torno a este problema entre las docentes), se logró una mejor recuperación y sistematización de lo discutido en los grupos. Para ello, se elaboraron preguntas que organizaron la discusión colectiva así como cuadros sinópticos para captar diferentes aspectos de lo intercambiado en los grupos. En esta instancia, se buscó orientar la reflexión a la identificación de los aspectos comunes de los trabajos de los/as estudiantes así como de los aprendizajes realizados a partir del diálogo y del entrometerse «en la cocina» o «trastienda» de la investigación de los restantes participantes (Sautu y Wainerman 1997). En este momento de la discusión grupal (al igual que en las clases teóricas), pudimos compartir nuestras propias experiencias y transmitir distintos «trucos» propios del oficio de hacer investigación cualitativa. En diferentes oportunidades, presentamos ejemplos de nuestras investigaciones. Si bien compartimos logros, fundamentalmente nuestras intervenciones apuntaban a identificar problemas, dudas, inquietudes y encrucijadas comunes así como las formas en que los enfrentamos. Aquí, siguiendo a Kvale (2006), socializamos nuestras experiencias de investigación con el propósito de visibilizar aquéllos dilemas que son comunes a todo investigador/a cualitativo/a y que, sin embargo, suponen decisiones únicas, no estandarizadas ni automáticas. El objetivo no era brindar recetas -lo cual sería imposiblesino explicitar el carácter singular de cada investigación.

${ }^{8}$ Éstos fueron distribuidos a los respectivos miembros de los grupos con antelación.

EMPIRIA. Revista de Metodología de Ciencias Sociales. N. ${ }^{\circ}$ 17, enero-junio, 2009, pp. 123-140. ISSN: 1139-5737 


\section{CONSIDERACIONES FINALES}

Este trabajo argumentó que la enseñanza del uso de entrevistas en investigación social requiere un despliegue de diferentes instancias pedagógicas que contribuyan a la adquisición de habilidades y saberes prácticos (propios de los oficios) y profesionales. En la primera sección presentamos el modelo de investigador/a cualitativo/a que orientó nuestra tarea docente. Desde nuestra perspectiva, la persona que investiga desde un enfoque cualitativo debe ser entendida como un profesional que lleva adelante una tarea con características similares a los oficios que se nutre también de la creatividad y la imaginación - similar a la que despliegan los artistas aunque con sus propias formas y criterios de comunicación. En la segunda sección, examinamos dos instancias pedagógicas - el uso de ejercicios y guías prácticas- que definimos como interview practica. Ambas instancias resultaron apropiadas para pensar la actividad de hacer una investigación con entrevistas, con flexibilidad para resolver y considerar cuestiones no previstas (los emergentes propios del campo) y con rigurosidad para hacerlo comunicable y sujeto a crítica. Estos criterios (comunicable y sujeto a crítica) son característicos de la investigación social. El carácter creativo de la profesión y oficio de hacer entrevistas adquiría más fuerza en cada una de las interview practica desplegadas en el taller. Fue posible poner en práctica habilidades reflexivas para pensar qué se estaba haciendo, desde qué perspectiva se lo hacía, así como para reformular ciertos componentes del diseño. Las diferentes instancias de interview practica agilizaron músculos intelectuales orientados a mayores niveles de reflexividad así como la imaginación sociológica y la creatividad.

Esta presentación de una instancia de enseñanza sin tropiezos no debe ocultar que a pesar de la excelente recepción, desarrollo y evaluación del curso, estudiantes y docentes reconocieron una debilidad central en el desarrollo del taller: la falta de tiempo para una discusión más profunda de las temáticas tratadas. Esta necesidad de más espacio para la reflexión y práctica, refleja que uno aprende a hacer investigación a partir de la ejercitación constante donde la guía de un/a «maestro/a» resulta útil.

En nuestro caso, la transferencia de la propia práctica resultó valiosa, sobre todo para señalar que la investigación lejos de ser un proceso lineal y puro, esta lleno de curvas y contracurvas, avances y retrocesos y eso es la investigación en todos los campos y las disciplinas. La investigación es un proceso activo de búsqueda de respuestas a nuestros interrogantes a través de caminos muy diversos. Enseñar a hacer investigación es presentar esa diversidad en acción, esto es, a partir de la realidad de lo investigado, con sus obstáculos, sus éxitos, sus aparentes fracasos y nuevas búsquedas para arribar a una investigación creíble, auténtica, rigurosa y creativa. Investigar cualitativamente es una puesta en práctica diaria de diversas modalidades de trabajo en función de cada situación concreta, registrando cada paso, cada decisión, cada resultado para hacer comunicable y sujetos a crítica nuestras interpretaciones y hallazgos.

EMPIRIA. Revista de Metodología de Ciencias Sociales. N.o 17, enero-junio, 2009, pp. 123-140. ISSN: 1139-5737 


\section{BIBLIOGRAFÍA}

BECKER, H. S. (1998). Tricks of the trade. How you think about your research while you're doing it. Chicago: The University of Chicago Press.

BOURDIEU, P. y WACQUANT, L. J. D. (1995). Respuestas por una antropología reflexiva. México: Grijalbo.

BRYMAN, A. (2000). Quantity and quality in social research. London: Routledge.

Coffey, A. 1999. The ethnographic self: fieldwork and the representation of identity. London: Sage.

DENZIN, N. y LINCOLN, Y. S. (eds.) (2000). Handbook of qualitative research. London: Sage Publications.

GLASER, B. G., y A. L. STRAUSS. (1967). The discovery of grounded theory. Strategies for qualitative research. Aldine de Gruyter.

GUBER, R. (2004). El salvaje metropolitano. Reconstrucción del conocimiento social en el trabajo de campo. Buenos Aires: Paidós.

GUBRIUM, J. F. y Holstein, J. A. (2002). Handbook of interview research: context \& method. Thousand Oaks: Sage Publications.

HAMMERSLEY, M. (1992) What's wrong with ethnography? London: Routledge.

HAMMERSLEY, M. (2004). 'Teaching qualitative method: craft, profession, or bricolage?' en C. Seale, G. Gobo, J. F. Gubrium y D. Silverman (eds.), Qualitative research in practice. London: SAGE Publications.

HAMMERSLEY, M. y ATKINSON, P. (1995). Ethnography: principles in practice. London: Routledge.

HERTZ, R. (1997). 'Introduction: reflexivity and voice', en R. Hertz (ed.), Reflexivity and voice. London: Sage Publications, Inc.

JENKINS, R. (1995) 'Social Skills, Social Research Skills, Sociological Skills: Teaching Reflexivity?' Teaching Sociology, 23(1): 16-27.

KVALE, S. (1996). Interviews. An introduction to qualitative research interviewing. London: Sage Publications.

KVALE, S. (2006).»Interviewing between mwthod and craft». Denmark: Department of Psychology, University of Aarhus.

LAHIRE, BERNARD. (2006). El espíritu sociológico. Manantial.

MACBETH, D. (2001). 'On «reflexivity» in qualitative research: two readings, and a third', Qualitative Inquiry, 7: 35-68.

MATON, K. (2003). 'Reflexivity, relationism \& research. Pierre Bourdieu and the epistemic conditions of social scientific knowledge', space \& culture, 6(1): 52-65.

MEO, ANALIA. (2009). «Consideraciones éticas en investigación social.» en La voz de los otros. El uso de la entrevista en investigación, en A. Meo, y A. Navarro. Omicron System.

MAXWELL, J. (1996). ‘A model for qualitative research design', en J. Maxwell (ed.), Qualitative research design and interactive approach. Thousand Oaks: Sage Publications.

MILLS, C. W. (1994). La imaginación sociológica. México: Fondo de Cultura Económica.

NISBET, R. A. (1963). 'The rediscovery of sociology: some straws in the wind. Sociology as an art form', en Stein y A. J. Vidich (eds.), Sociology on trial. Englewwod Cliffs, NJ: Prentice-Hall.

PILLOW, W. S. (2003). 'Confession, cahtarsis, or cure? Rethinking the uses of reflexivity 
as methodological power in qualitative research', Qualitative Studies in Education, 16(2): 175-96.

REAY, D. (1996). 'Dealing with difficult differences: reflexivity and social class in feminist research', Feminism \& Psychology, 6: 443-56.

SAUTU, R. (1997). 'Acerca de qué es y no es Investigación Científica en Ciencias Sociales', en R. Sautu y C. Wainerman (comp.), La trastienda de la investigación. Buenos Aires: Editorial Universidad de Belgrano.

SAUTU, R. y C. WAINERMAN (1997). La trastienda de la investigación. Buenos Aires: Editorial Universidad de Belgrano.

SCHWANDT, T. A. (2000). 'Three epistemological stances for qualitative inquiry: interpretativism, hermeneutics, and social constructionism', en N. Denzin y Y. Lincoln (eds.), Handbook of qualitative research. Thousand Oaks: Sage Publications Inc.

VASILACHIS DE GIALDINO, I. (ed.) (2007). Estrategias de investigación cualitativa. Gedisa: Buenos Aires.

VIDICH, A. J. y LYMAN, S. M. (1994). 'Qualitative methods: their history in sociology and anthropology', en N. Denzin y Y. Lincoln (eds.), Handbook of qualitative research. London: Sage Publications.

\section{RESUMEN}

A partir del análisis de un taller sobre el uso de entrevistas en investigación desarrollado en doctorado de Ciencias Sociales de la Universidad de Buenos Aires (Argentina), este artículo argumenta que la enseñanza del uso de esta técnica exige el despliegue de variadas instancias pedagógicas o interview practica (Kvale 2006) capaces de contribuir a la apropiación de los aspectos profesionales, creativos y propios del oficio de hacer investigación cualitativa. La primera parte examina cuál es la concepción del rol de la persona que investiga cualitativamente, la cual subyació a la selección de los contenidos y el diseño pedagógico del taller. Se argumenta que el trabajo de los investigadores cualitativos debe ser entendido como una profesión creativa con una dimensión propia de los oficios La segunda sección analiza las características generales del taller y de su población así como dos tipos de interview practica utilizados: los ejercicios individuales y las guías de discusión. Se examina cómo estas instancias pedagógicas contribuyeron a la apropiación práctica, por parte de los/as estudiantes, de un habitus de investigación que promueve disposiciones y orientaciones a la acción reflexivas, éticas, profesionales y creativas.

\section{PALABRAS CLAVE}

Investigación cualitativa, docencia, posgrado, entrevistas 


\begin{abstract}
This article examines a module on interviewing in social sciences at the Doctoral programme in Social Sciences of the University of Buenos Aires (Argentina). It argues that teaching this research technique demands the use of a variety of pedagogic devices or interview practica (Kvale 2006). They facilitate the acquisition of the crafty, professional and creative aspects of qualitative research. Firstly, this paper examines the nature of the role of the qualitative researcher that underpinned the selection of contents and the pedagogic organisation of the module. It argues that the job of qualitative researchers should be understood as a profession with crafty and creative dimensions. Secondly, this article depicts the module, its population and two types of interview practice: individual exercises and discussion guides. These pedagogic devices are examined. Evidence of their contribution to the practical acquisition of a research habitus is presented. This habitus instils reflexive, professional, ethical and creative attitudes and dispositions in doctoral students, crucial for their professional development.
\end{abstract}

\title{
KEYWORDS
}

Qualitative research, teaching, postgraduate, interview 